\title{
Hot Carriers in CVD-Grown Graphene Device with a Top h-BN Layer
}

\section{Chuang $\left(\mathbb{D},{ }^{1,2}\right.$ M. Mineharu, ${ }^{1}$ N. Matsumoto, ${ }^{1}$ M. Matsunaga, ${ }^{1}$ C.-W. Liu, ${ }^{3}$ B.-Y. Wu, ${ }^{3}$ Gil-Ho Kim, ${ }^{4}$ L.-H. Lin, ${ }^{5}$ Y. Ochiai, ${ }^{1}$ K. Watanabe, ${ }^{6}$ T. Taniguchi, ${ }^{6}$ C.-T. Liang $\left.{ }^{(D}\right)^{3}$ and N. Aoki ${ }^{1}{ }^{1}$}

${ }^{1}$ Department of Materials Science, Chiba University, Chiba 263-8522, Japan

${ }^{2}$ Institute of Atomic and Molecular Sciences, Academia Sinica, Taipei 10617, Taiwan

${ }^{3}$ Graduate Institute of Applied Physics, National Taiwan University, Taipei 10617, Taiwan

${ }^{4}$ School of Electronic and Electrical Engineering, Sungkyunkwan University, Suwon 16419, Republic of Korea

${ }^{5}$ Department of Electrophysics, National Chiayi University, Chiayi 600, Taiwan

${ }^{6}$ Advanced Materials Laboratory, National Institute for Materials Science, 1-1 Namiki, Tsukuba 305-0044, Japan

Correspondence should be addressed to C.-T. Liang; ctliang@phys.ntu.edu.tw and N. Aoki; n-aoki@faculty.chiba-u.jp

Received 18 January 2018; Accepted 20 March 2018; Published 14 May 2018

Academic Editor: Stefano Bellucci

Copyright $(0) 2018$ C. Chuang et al. This is an open access article distributed under the Creative Commons Attribution License, which permits unrestricted use, distribution, and reproduction in any medium, provided the original work is properly cited.

\begin{abstract}
We investigate the energy relaxation of hot carriers in a CVD-grown graphene device with a top $h$-BN layer by driving the devices into the nonequilibrium regime. By using the magnetic field dependent conductance fluctuations of our graphene device as a self-thermometer, we can determine the effective carrier temperature $T_{\mathrm{e}}$ at various driving currents $I$ while keeping the lattice temperature $T_{\mathrm{L}}$ fixed. Interestingly, it is found that $T_{\mathrm{e}}$ is proportional to $I$, indicating little electron-phonon scattering in our device. Furthermore the average rate of energy loss per carrier $P_{\mathrm{e}}$ is proportional to $\left(T_{\mathrm{e}}{ }^{2}-T_{\mathrm{L}}{ }^{2}\right)$, suggesting the heat diffusion rather than acoustic phonon processes in our system. The long energy relaxation times due to the weak electron-phonon coupling in CVD graphene capped with h-BN layer as well as in exfoliated multilayer graphene can find applications in hot carrier graphene-based devices.
\end{abstract}

\section{Introduction}

Recently, researchers in the graphene community are much interested in hot carriers in graphene-based systems since they determine the performance of high-power and highfrequency electronics, thermal management of electronic devices, optoelectronic devices, the quantum Hall metrology, and bolometric detectors [1-6]. Most of the hot carrier graphene devices in the high carrier density limit (the BlochGruneisen temperature $\left(T_{\mathrm{BG}}\right)>$ the lattice temperature $\left(T_{\mathrm{L}}\right)$ ) show the dominant cooling power from a weak coupling of carriers to acoustic phonon processes [7-10], which is represented by a heat flow power law equation $P=\Sigma\left(T_{\mathrm{e}}^{\delta}-T_{\mathrm{L}}^{\delta}\right)[11]$ where $\delta=4$ is a characteristic exponent, $T_{\mathrm{e}}$ is the carrier temperature, $T_{\mathrm{L}}$ is the lattice temperature, and $\Sigma$ is the coupling constant. Furthermore, disordered-enhanced properties in hot carrier graphene devices revealed the supercollision cooling processes, where $\delta=3$ [12-14]. Recently, hexagonal Boron Nitride (h-BN) bottom substrate can be a great heat drained material for disordered graphene so as to extremely reduce the carrier-phonon scattering via Wiedemann-Franz law heat diffusion, which is $\delta=2$ [15-17]. However, the carriers of energy relaxation in such $\mathrm{h}$-BN/disordered graphene systems with less substrate phonon interactions following the Wiedemann-Franz law heat diffusion are rarely studied. A key parameter for discussing the cooling process is the energy relaxation time $\left(\tau_{\epsilon}\right)$, the characteristic time when the thermal energy is lost by the carriers [18-20].

To date, CVD-grown graphene appears to be a good candidate for large-scale graphene-based applications. However, as such a system may not be air stable, it is highly desirable to cap the CVD graphene with an inert layer so 


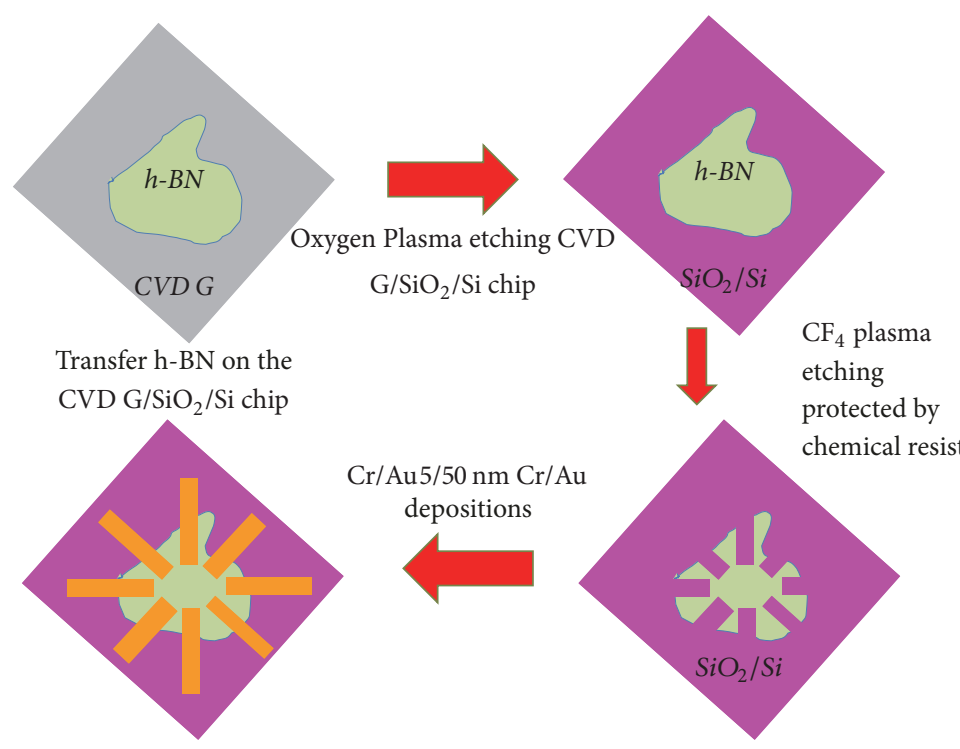

(a)

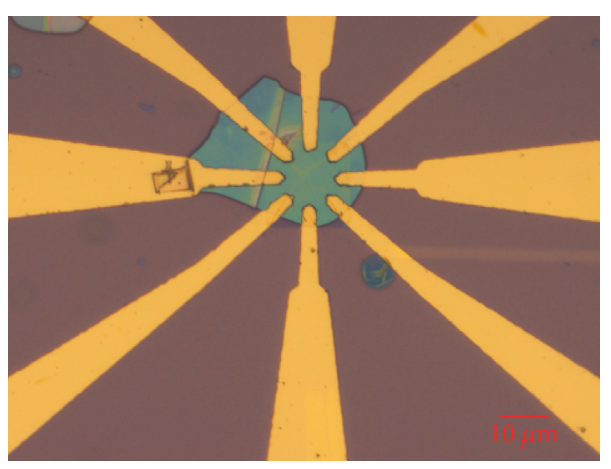

(b)

FIGURE 1: (a) Schematic fabrication diagram of a top h-BN/CVD graphene device. (b) The top h-BN/CVD graphene device (sample A) with a scale bar of $10 \mu \mathrm{m}$.

as to experimentally realize stable, large-scale, and scalable devices. To this end, we have prepared CVD graphene with a top h-BN layer and studied the hot carrier effects in such a device. In this report, we studied the conductance fluctuations and hot carrier effects using a large measurement current (I) so as to raise the effective $T_{\mathrm{e}}$ above $T_{\mathrm{L}}$ on $\mathrm{h}$ $\mathrm{BN} / \mathrm{CVD}$ graphene and multilayer exfoliated graphene due to their disordered properties. Based on the self-thermometer linear properties between $T_{\mathrm{e}}$ and $I$ due to WiedemannFranz law heat diffusion, we can investigate energy relaxation cooling processes under high carrier density limit $\left(T_{B G}>T_{L}\right)$ between the h-BN/CVD graphene and multilayer exfoliated graphene for future graphene-based applications. By determining $\tau_{\epsilon}$ from $T_{\mathrm{e}}$ and input power $P_{\mathrm{e}}$, we can find that $\tau_{\epsilon}$ of the CVD graphene with a top h-BN layer is almost two orders of magnitude longer than those in exfoliated pristine monolayer/bilayer graphene. Such a result may find applications in hot carrier graphene-based transistors as a result of the weak electron-phonon coupling.

\section{Experimental Section}

2.1. Preparation of the Samples. As shown in the schematic diagram in Figure 1(a), we used the scotch tape method to mechanically exfoliate high purity and homogenous h-BN crystals synthesized by high pressure techniques [21] and transferred them by Gel-Pak polymer using the viscoelastic effect [22] on commercial chemical vapor deposition (CVD) graphene $/ 285 \mathrm{~nm} \mathrm{SiO}_{2} / \mathrm{Si}$ substrate [23]. The CVD graphene region outside $h-B N$ sheet was etched by oxygen plasma so as to confine to our CVD graphene region under the $h-B N$ sheet. $\mathrm{CF}_{4}$ gas was used to etch h-BN sheet protected by photoresist for 8 terminals and $\mathrm{Cr} / \mathrm{Au}$ metal depositions as shown in Figure 1(b).
2.2. Electrical Measurements. An ac driving current from lock-in amplifiers passed into the source and drain contacts through the graphene devices for Hall-bar measurements. The magnetoresistance was measured in a $\mathrm{He}^{3}$ cryostat equipped with a superconducting magnet.

\section{Results and Discussion}

Figure 2(a) shows the longitudinal resistivity $\rho_{x x}$ as a function of magnetic field with fixed current $I=20 \mathrm{nA}$ at different temperatures that are equivalent to $T_{\mathrm{L}}$. The conductance fluctuations are observed, and they decrease as $T_{\mathrm{L}}$ increase from $T_{\mathrm{L}}=0.32$ to $50 \mathrm{~K}$, which are typical properties in disordered mesoscopic graphene [24-27]. Figure 2(a) inset shows the corresponding Hall resistivity at $T_{\mathrm{L}}=0.32 \mathrm{~K}$, which can calculate the carrier density $n_{\mathrm{A}}=3.5 \times 10^{12} \mathrm{~cm}^{-2}$ from the Hall slope and Hall mobility $\mu=2092 \mathrm{~cm}^{2} /$ Vs from carrier density and zero field resistivity. Figure 2 (b) shows the results of $\rho_{x x}$ at various driving currents $I$ from $I=20 \mathrm{nA}$ to $30000 \mathrm{nA}$ at a fixed $T_{\mathrm{L}}$ of $0.32 \mathrm{~K}$, which reveals similar conductance fluctuation characteristics by current heating in the nonequilibrium regime due to the hot carrier effects in disordered two-dimensional systems [18, 28-31].

By using a conductance fluctuations-based thermometer between $\rho_{x x}(I)$ and $\rho_{x x}(T)$, we are able to reveal the clear conductance variations for lattice temperature and current dependence between $B=0.5$ and $3 \mathrm{~T}$ by subtracting a smooth background that avoids the zero field weak localization peak and high field Shubnikov-de Haas-like oscillations as shown in Figures 3(a) and 3(b) [13]. Hence, we determine the root mean square (RMS) conductance fluctuation $\left(\delta g_{\mathrm{rms}}\right.$, in units of $\mathrm{e}^{2} / \mathrm{h}$ ) for every $\delta g_{\mathrm{rms}}\left(T_{\mathrm{L}}\right)$ and $\delta g_{\mathrm{rms}}(I)$ data set as a common graph (see Supporting Information (available 


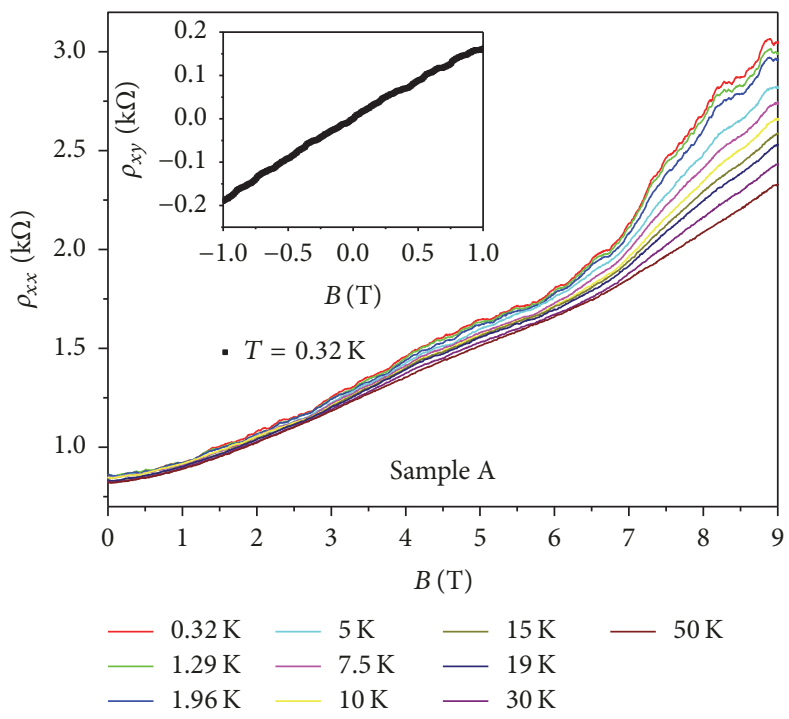

(a)

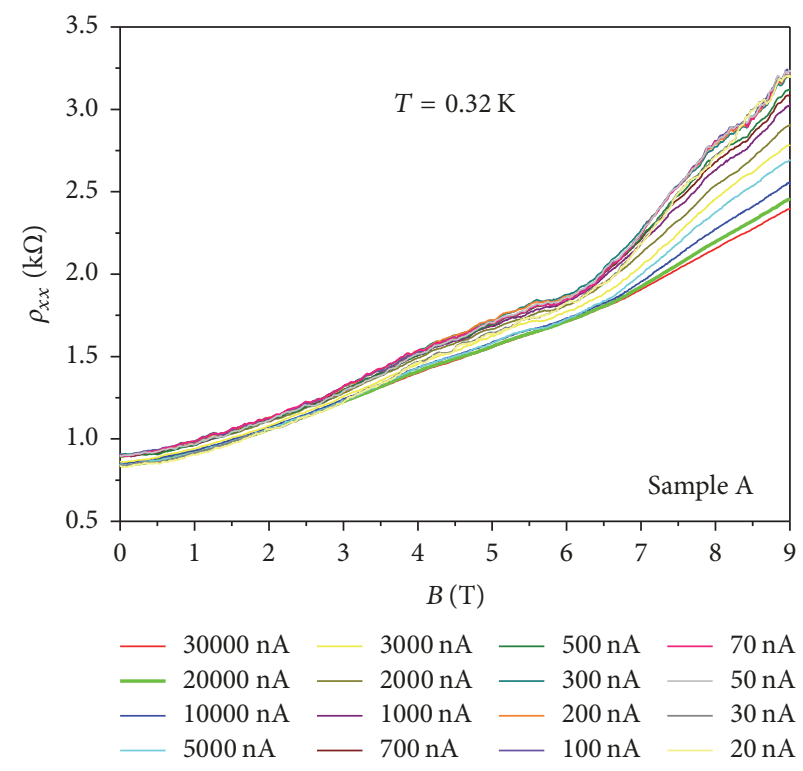

(b)

Figure 2: (a) Magnetoresistivity $\rho_{x x}(B)$ at various $T_{\mathrm{L}}$ for sample A. The inset shows the Hall resistivity $\rho_{x y}(B)$ at $T_{\mathrm{L}}=0.32 \mathrm{~K}$. (b) Magnetoresistivity $\rho_{x x}(B)$ at various driving currents $I$ at fixed $T_{\mathrm{L}}=0.32 \mathrm{~K}$ for sample A.

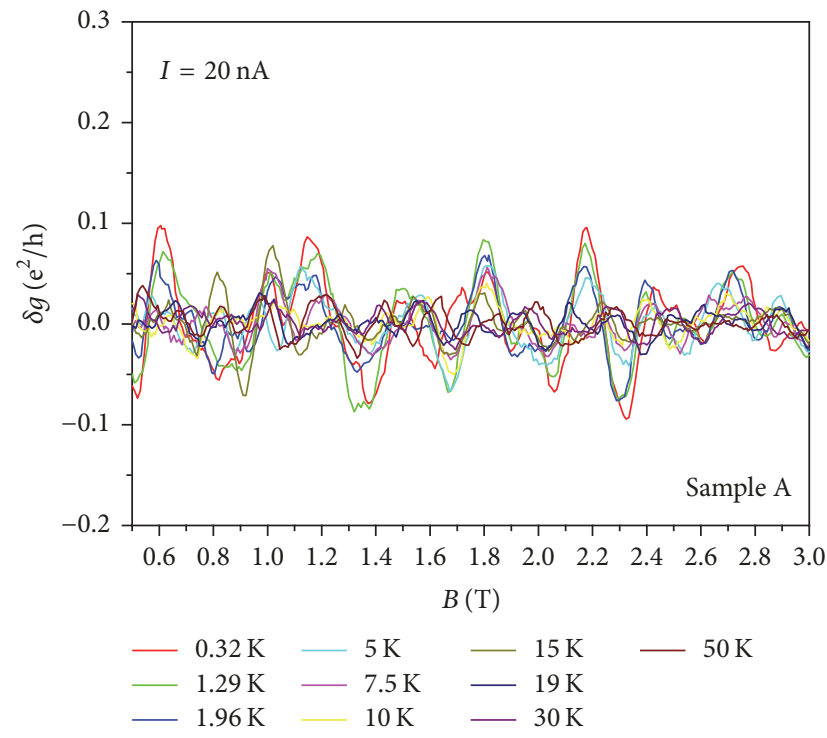

(a)

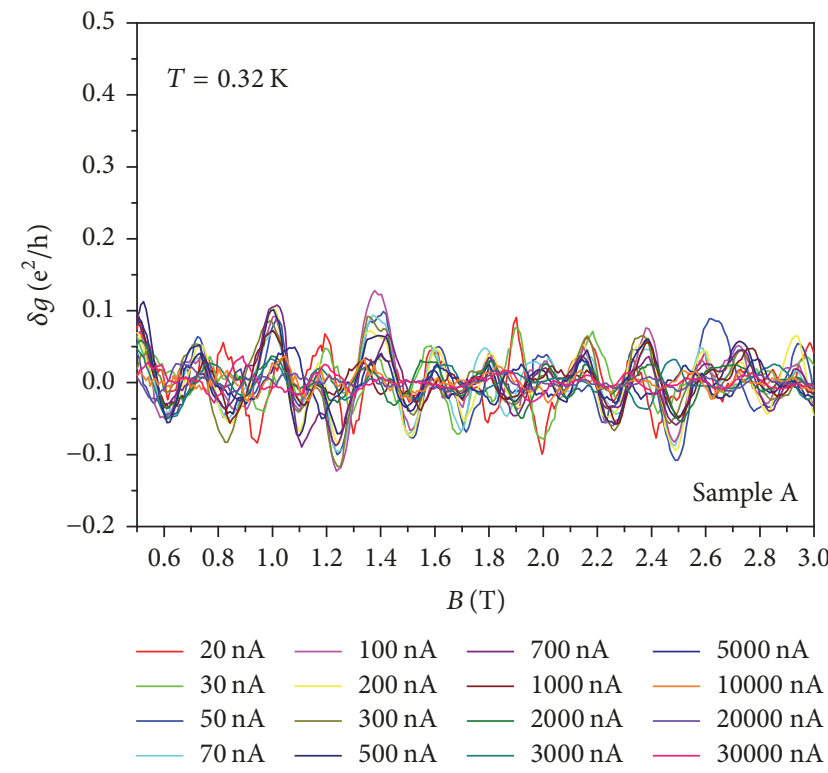

(b)

Figure 3: (a) Conductance fluctuations in sample A as a function of magnetic field at various lattice temperatures with fixed $I=20 \mathrm{nA}$. (b) Conductance fluctuations in sample A as a function of magnetic field at various driving currents for $T_{\mathrm{L}}=0.32 \mathrm{~K}$.

here)) by averaging conductance fluctuations over the range of $B$ so as to assign an effective $T_{\mathrm{e}}$ to the driving current as shown in Figure $4(\mathrm{a})[13,18]$. Interestingly, $T_{\mathrm{e}}$ shows a linear dependence on $I$. According to the seminal work done by Baker and coworkers about the energy transfer between carriers and the lattice [31], the following relation can be found:

$$
T_{\mathrm{e}} \propto I^{\alpha}
$$

where $\alpha=2 /(p+2)$ and $p$ is the exponent for the inelastic scattering rate $\tau_{\text {in }}{ }^{-1} \propto T^{p}$. Therefore, Figure 4(a) shows that $T_{\mathrm{e}}(I)$ of our sample A follows (1) with $p \approx 0$ and $\alpha \approx 1$, which suggested little carrier-phonon scattering in two-dimensional material heterostructure systems [26]. Furthermore, one would be interested in whether the heat dissipation was transferred by another mechanism rather than carrier-phonon scattering. Under the low-temperature limit $\left(T_{\mathrm{L}}<T_{\mathrm{BG}}\right), T_{\mathrm{BG}}=2 \hbar s k_{\mathrm{F}} / k_{\mathrm{B}}$, where $s$ is the sound 


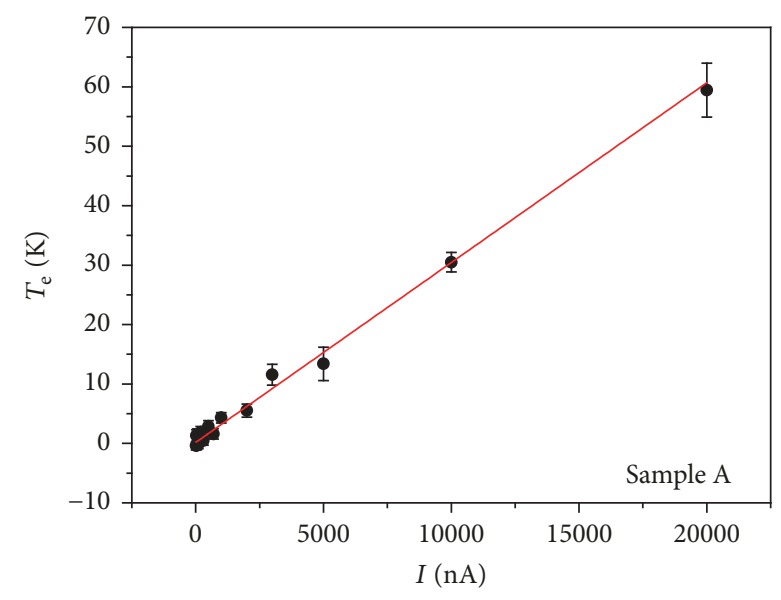

(a)

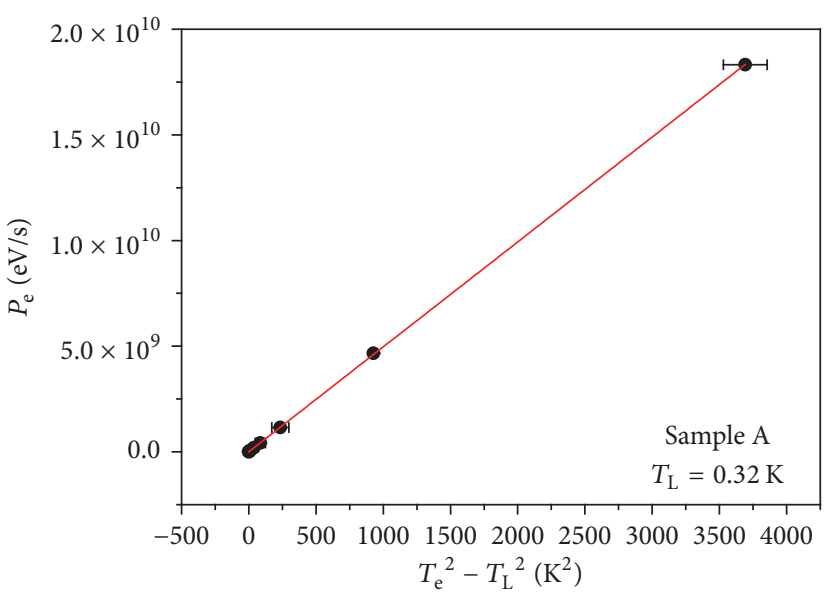

(b)

Figure 4: (a) Effective $T_{\mathrm{e}}$ versus driving current $I$ in sample A. (b) $P_{\mathrm{e}}$ as a function of $\left(T_{\mathrm{e}}{ }^{2}-T_{\mathrm{L}}{ }^{2}\right)$ for sample $\mathrm{A}$ and at $T_{\mathrm{L}}=0.32 \mathrm{~K}$.

velocity and $k_{\mathrm{F}}$ is the Fermi radius. For graphene systems ( $s$ $\left.=2.1 \times 10^{4} \mathrm{~ms}^{-1}\right), T_{\mathrm{BG}} \sim 54 n^{1 / 2} \mathrm{~K} \cong 93.4 \mathrm{~K}$ with the carrier density $n=3.5$ in units of $10^{12} \mathrm{~cm}^{-2}$ in sample A $[13,32]$. The energy loss $\left(P_{\mathrm{e}}\right)$, the average rate of energy loss per carrier, is usually expressed to the carrier and lattice temperatures as

$$
P_{\mathrm{e}}=A\left(T_{\mathrm{e}}^{\beta}-T_{\mathrm{L}}^{\beta}\right) \text {, }
$$

with a constant $A$ and a characteristic exponent $\beta$. In general graphene systems, $\beta \approx 3$ suggests the supercollision cooling mechanism in disordered systems $[13,18,33]$ and $\beta \approx$ 4 suggests the two-dimensional acoustic phonon cooling processes in clean systems $[34,35]$. Particularly, $\beta \approx 2$, the heat diffusion described by the Wiedemann-Franz law, was found in graphene on bottom h-BN substrate systems, where bottom h-BN substrate acts as a thermal conduction layer, effectively reducing the electron-phonon coupling [1416]. Such interesting bottom h-BN substrate underneath graphene can change graphene heat transport mechanism from electron-phonon interactions $(\beta \approx 3 \sim 4)$ to heat diffusion $(\beta \approx 2)$. One might be interested in what heat transport mechanism will be in encapsulated graphene between bottom insulating $\mathrm{SiO}_{2}$ and top $h-B N$ sheet as our sample A rather than traditional graphene on $\mathrm{h}-\mathrm{BN}$ substrate [14-16]. Based on the work of Baker et al. [31], $\beta=p+$ 2 , which suggested $\beta$ in sample $A$ should be close to 2 since the linear relation between $T_{\mathrm{e}}$ and $I$ is $p \approx 0(1=\alpha=$ $2 /(p+2))$. Apparently, our results in sample A are highly consistent with this speculation for $\beta \approx 2$ as shown in Figure 4(b), where $P_{\mathrm{e}}=I^{2} R_{x x} / n W L$ ( $W$ and $L$ are the width and length of our sample $\mathrm{A}$, resp.). Interestingly, the heat transfer mechanism in our sample A with top h-BN sheet on graphene/ $/ \mathrm{SiO}_{2}$ was dominated by heat diffusion $(\beta \approx 2)$, which is the same as traditional graphene/h-BN substrate devices [14-16]. Such interesting results suggest that the top h-BN sheet coupled with graphene is strong heat transfer medium in comparison with other coupled materials, like $\mathrm{SiO}_{2}$ or air. Also, such structures in the top h-BN sheet on graphene not only protected graphene without air molecular adsorbing doping [2-36], but also screened graphene from electrostatic force $[37,38]$, a great advancement for graphenebased devices.

In order to further discuss the linear relation between $T_{\mathrm{e}}$ and $I$ that indicates little carrier-phonon scattering in our sample $\mathrm{A}$, we fabricate multilayer exfoliated graphene (sample B) that showed the same linear relation between $T_{\mathrm{e}}$ and $I$ by zero field resistance as a thermometer and conductance fluctuations due to its disordered property as in our previous reports [23-26]. Again, we are able to measure the conductance fluctuations for temperature and current dependence as shown in Figure 5(a) and inset [13]. Consequently, we found the linear relation between $T_{\mathrm{e}}$ and $I$ by utilizing $\delta g_{\mathrm{rms}}\left(T_{\mathrm{L}}\right)$ and $\delta g_{\mathrm{rms}}(I)$ as a thermometer for determining $T_{\mathrm{e}}$ (see Supporting Information) [13] as shown in the inset of Figure 5(b), which suggested $p \approx 0$ and $\beta \approx 2$. Under the low-temperature limit $\left(T_{\mathrm{L}}<T_{\mathrm{BG}}\right)$, $T_{\mathrm{BG}} \sim 54 n^{1 / 2} \mathrm{~K} \cong 188.6 \mathrm{~K}$ with the carrier density $n=$ 12.2 in units of $10^{12} \mathrm{~cm}^{-2}$ in sample B $[13,32]$. Consistently, $P_{\mathrm{e}}$ is proportional to $\left(T_{\mathrm{e}}^{2}-T_{\mathrm{L}}^{2}\right)$ in sample $\mathrm{B}$ as shown in Figure 5(b), which belonged to Wiedemann-Franz law heat diffusion and corresponded to the transport situation for little carrier-phonon scattering for $\alpha \approx 1[7,26]$. Interestingly, the multilayer exfoliated graphene (sample B) has the same heat transfer mechanism $(\beta \approx 2)$ as the h-BN/graphene (sample A) systems [14-16].

Although the heat transfer mechanism of multilayer exfoliated graphene is the same as h-BN/CVD graphene for heat diffusion $(\beta \approx 2)$, the energy relaxation time can describe this cooling process about the typical time on which energy is lost from carriers $[39,40]$. Therefore, the energy relaxation time can be expressed by

$$
P_{\mathrm{e}}=\frac{k_{\mathrm{B}}\left(T_{\mathrm{e}}-T_{\mathrm{L}}\right)}{\tau_{\epsilon}} .
$$

Figure 6 compares the energy relaxation time $\tau_{\epsilon}$ between the h-BN/CVD graphene (sample A) and the multilayer 


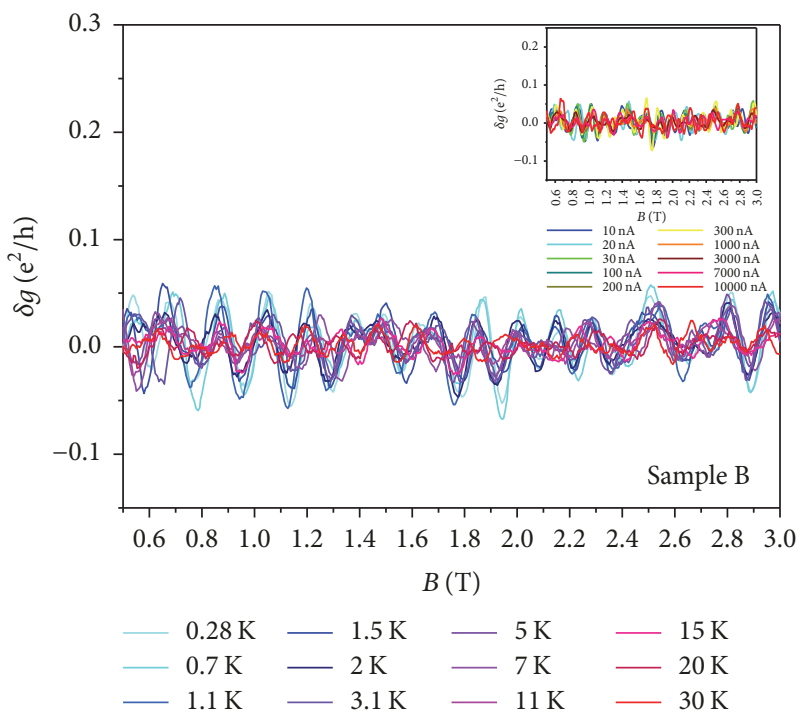

(a)

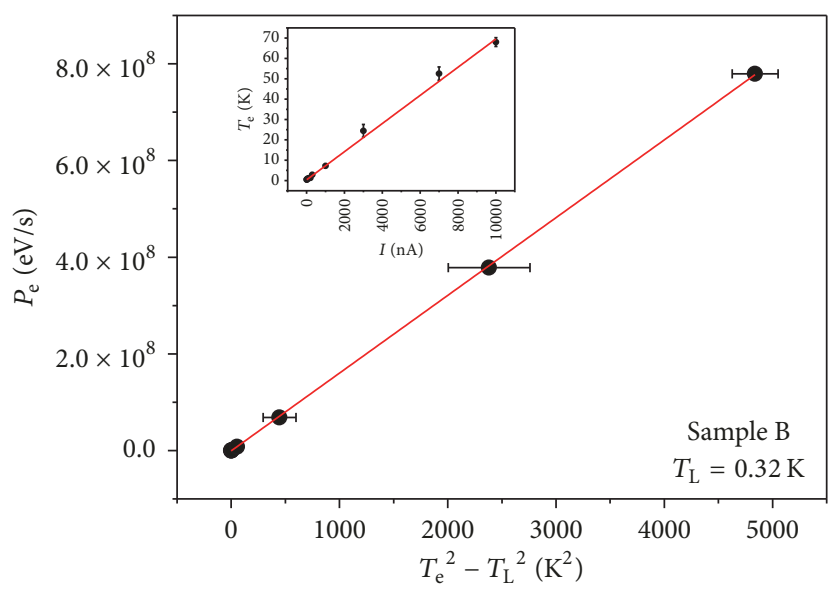

(b)

FIGURE 5: (a) Conductance fluctuations in sample B as a function of magnetic field at various $T_{\mathrm{L}}$ with fixed $I=20 \mathrm{nA}$. The inset shows conductance fluctuations in sample $\mathrm{B}$ as a function of magnetic field with various driving currents at fixed $T_{\mathrm{L}}=0.32 \mathrm{~K}$. (b) $P_{\mathrm{e}}$ as a function of $\left(T_{\mathrm{e}}{ }^{2}-T_{\mathrm{L}}{ }^{2}\right)$ for sample $\mathrm{B}$ and at $T_{\mathrm{L}}=0.32 \mathrm{~K}$. The inset shows effective $T_{\mathrm{e}}$ versus driving current $I$ in sample $\mathrm{B}$.

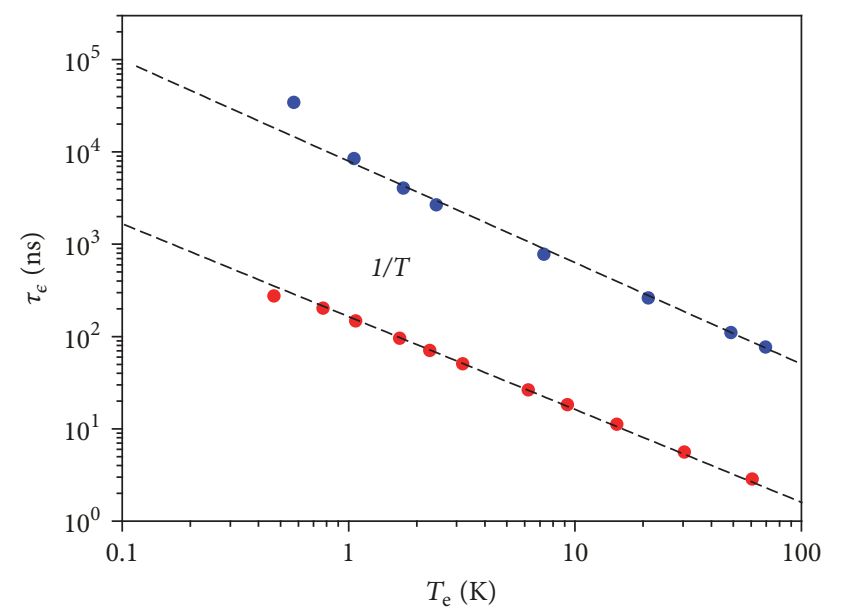

- Multi-layer graphene

- H-BN/CVD graphene

Figure 6: (a) Comparison of $\tau_{\epsilon}\left(T_{\mathrm{e}}\right)$ versus $T_{\mathrm{e}}$ on a log-log scale for sample A (h-BN/CVD graphene) and sample B (multilayer graphene).

exfoliated graphene (sample B). Interestingly, we could obviously find that $\tau_{\epsilon}$ of h-BN/CVD graphene (sample A) is about two orders of magnitude faster than that of multilayer graphene (sample B). Both of these two samples are the same $n$-type of carrier density and far from the Dirac point, which cannot be ascribed to the fast energy relaxation of hot carriers near the Dirac point of graphene [18]. These results suggest that the carrier-phonon scattering is absent in the multilayer graphene and the h-BN/graphene. The extremely long energy relaxation times in both devices (at least two orders of magnitudes longer than those in pristine exfoliated graphene $[27,28]$ and graphene on $\mathrm{SiC}[30,34])$ can be advantageous for applications in graphene-based hot carrier transistors [41] since carriers can maintain their high kinetic energy (and hence the high effective temperature) with a relatively low driving current.

\section{Conclusion}

We have studied conductance fluctuations and hot carrier effects caused by current heating on h-BN/CVD graphene and multilayer graphene as a self-thermometer. It has been shown that $T_{\mathrm{e}}\left(P_{\mathrm{e}}\right)$ is linearly proportional to $I\left(T_{\mathrm{e}}{ }^{2}-T_{\mathrm{L}}{ }^{2}\right)$ in both of the disordered graphene devices, suggesting the little electron-phonon scattering and heat diffusion due to Wiedemann-Franz law. The extremely long energy relaxation time may find applications in graphene-based hot carrier devices. Such a result may be useful for other material systems $[42,43]$.

\section{Data Availability}

The data used to support the findings of this study are available from the corresponding author upon request

\section{Conflicts of Interest}

The authors declare that they have no conflicts of interest.

\section{Acknowledgments}

As an International Research Fellow of the Japan Society for the Promotion of Science (JSPS), C. Chuang acknowledges 
a JSPS Postdoctoral Fellowship and the MOST and Institute of Atomic and Molecular Sciences, Academia Sinica, for a postdoctoral fellowship under the Contract no. MOST 1062811-M-001-164. C.-T. Liang would like to acknowledge the Ministry of Science and Technology (MOST), Taiwan, for financial support (Grant no. MOST 105-2119-M-002-048MY3). N. Aoki acknowledges funding from JSPS KAKENHI (Grant no. JP16H00899 on Innovative Areas "Science of Atomic Layers").

\section{Supplementary Materials}

This includes the method of determining the effective electron temperature using amplitudes of the observed conductance fluctuations. (Supplementary Materials)

\section{References}

[1] X. Du, D. E. Prober, H. Vora, and C. B. Mckitterick, "Graphenebased bolometers," Graphene 2D Mater, vol. 1, 2014.

[2] J. Huang, J. A. Alexander-Webber, T. J. B. M. Janssen et al., "Hot carrier relaxation of Dirac fermions in bilayer epitaxial graphene," Journal of Physics: Condensed Matter, vol. 27, Article ID 164202, 2015.

[3] C. Voisin and B. Plaçais, "Hot carriers in graphene," Journal of Physics: Condensed Matter, vol. 27, Article ID 160301, 2015.

[4] A. Tzalenchuk, S. Lara-Avila, A. Kalaboukhov et al., "Towards a quantum resistance standard based on epitaxial graphene," Nature Nanotechnology, vol. 5, p. 186, 2010.

[5] A. E. Fatimy, R. L. Myers-Ward, A. K. Boyd, K. M. Daniels, D. K. Gaskill, and P. Barbara, "Epitaxial graphene quantum dots for high-performance terahertz bolometers," Nature Nanotechnology, vol. 11, p. 335, 2016.

[6] Y. Yang, G. Cheng, P. Mende et al., "Epitaxial graphene homogeneity and quantum Hall effect in millimeter-scale devices," Carbon, vol. 115, pp. 229-236, 2017.

[7] R. Bistritzer and A. H. MacDonald, "Electronic cooling in graphene," Physical Review Letters, vol. 102, Article ID 206410, 2009.

[8] S. Kubakaddi, "Interaction of massless Dirac electrons with acoustic phonons in graphene at low temperatures," Physical Review Journals, vol. 79, Article ID 075417, 2009.

[9] W. K. Tse and S. Das Sarma, Physical Review B, vol. 79, Article ID 235406, 2009.

[10] J. K. Viljas and T. T. Heikkilä, "Electron-phonon heat transfer in monolayer and bilayer graphene," Physical Review B: Condensed Matter and Materials Physics, vol. 81, no. 24, 2010.

[11] F. Giazotto, T. T. Heikkilä, A. Luukanen, A. M. Savin, and J. P. Pekola, "Opportunities for mesoscopics in thermometry and refrigeration: Physics and applications," Reviews of Modern Physics, vol. 78, p. 217, 2006.

[12] M. W. Graham, S.-F. Shi, D. C. Ralph, J. Park, and P. L. McEuen, "Photocurrent measurements of supercollision cooling in graphene," Nature Physics, vol. 9, p. 103, 2013.

[13] K. Kechedzhi, D. W. Horsell, F. V. Tikhonenko et al., "Quantum Transport Thermometry for Electrons in Graphene," Physical Review Letters, vol. 06680, 2009.

[14] J. C. W. Song, M. Y. Reizer, and L. S. Levitov, "DisorderAssisted Electron-Phonon Scattering and Cooling Pathways in
Graphene," Physical Review Letters, vol. 109, Article ID 106602, 2012.

[15] A. de Felice, A. E. Gümrükçüoğlu, and S. Mukohyama, "Hot Electron Cooling by Acoustic Phonons in Graphene," Physical Review Letters, vol. 109, Article ID 171101, 2012.

[16] Q. Han, T. Gao, R. Zhang et al., "Highly sensitive hot electron bolometer based on disordered graphene," Scientific Reports, vol. 3, p. 3533, 2013.

[17] W. Yang, S. Berthou, X. Lu et al., "A graphene Zener-Klein transistor cooled by a hyperbolic substrate," Nature Nanotechnology, vol. 1702, Article ID 02829, 2018.

[18] R. Somphonsane, H. Ramamoorthy, G. Bohra et al., "Fast energy relaxation of hot carriers near the dirac point of graphene," Nano Letters, vol. 13, no. 9, pp. 4305-4310, 2013.

[19] D. K. Ferry, Semiconductor Transport, Taylor \& Francis, London, 2000.

[20] S. M. Sze and M. K. Lee, Semiconductor Devices: Physics and Technology, John Wiley and Sons, John Wiley and Sons, Hoboken, NJ, USA, 3rd edition, 2012.

[21] K. Watanabe, T. Taniguchi, and H. Kanda, "Direct-bandgap properties and evidence for ultraviolet lasing of hexagonal boron nitride single crystal," Nature Materials, vol. 3, p. 404, 2004.

[22] A. Castellanos-Gomez, L. Vicarelli, E. Prada et al., "Isolation and characterization of few-layer black phosphorus," 2D Materials, vol. 1, no. 2, Article ID 025001, 2014.

[23] “Two Layers of CVD Graphene Film on SiO2/Si Wafer: 4 pack," https:/graphene-supermarket.com/CVD-Graphene-on-SiO2$\mathrm{Si} /$.

[24] C. Chuang, L.-H. Lin, N. Aoki et al., "Mesoscopic conductance fluctuations in multi-layer graphene," Applied Physics Letters, vol. 103, no. 4, Article ID 043117, 2013.

[25] C. Chuang, L.-H. Lin, N. Aoki et al., "Experimental evidence for direct insulator-quantum Hall transition in multi-layer grapheme," Nanoscale Research Letters, vol. 8, article 214, pp. 15, 2013.

[26] C. Chuang, T.-P. Woo, A. M. Mahjoub et al., "Weak localization and universal conductance fluctuations in multi-layer graphene," Current Applied Physics, vol. 14, no. 1, pp. 108-111, 2014.

[27] J. Kim, S. Park, M. Ju, and C. Jeong, "Characteristics of carrier collection of nanopillar-patterned silicon solar cells," Journal of Nanoscience and Nanotechnology, vol. 15, no. 3, pp. 2400-2403, 2015.

[28] G. Bohra, R. Somphonsane, N. Aoki et al., "Nonergodicity and microscopic symmetry breaking of the conductance fluctuations in disordered mesoscopic graphene," Physical Review B: Condensed Matter and Materials Physics, vol. 86, no. 16, 2012.

[29] H. Ramamoorthy, R. Somphonsane, G. He, D. K. Ferry, and J. P. Bird, "Reversing hot-carrier energy-relaxation in graphene with a magnetic field," Applied Physics Letters, vol. 104, no. 19, p. 193115, 2014.

[30] J. J. Lin and J. P. Bird, "Recent experimental studies of electron dephasing in metal and semiconductor mesoscopic structures," Journal of Physics: Condensed Matter, vol. 14, p. R501, 2002.

[31] A. M. Baker, J. A. Alexander-Webber, T. Altebaeumer et al., "Energy loss rates of hot Dirac fermions in epitaxial, exfoliated, and CVD graphene," Physical Review B: Condensed Matter and Materials Physics, vol. 87, no. 4, 2013. 
[32] A. K. Wennberg, S. N. Ytterboe, C. M. Gould, H. M. Bozler, J. Klem, and H. Morkoç, "Electron heating in a multiplequantum-well structure below $1 \mathrm{~K}$," Physical Review B: Condensed Matter and Materials Physics, vol. 34, no. 6, pp. 44094411, 1986.

[33] A. C. Betz, S. H. Jhang, E. Pallecchi et al., "Supercollision cooling in undoped graphene," Nature Physics, vol. 9, no. 2, pp. 109-112, 2013.

[34] M. W. Graham, S. Shi, D. C. Ralph, J. Park, and P. L. McEuen, "Photocurrent measurements of supercollision cooling in graphene," Nature Physics, vol. 9, no. 2, pp. 103-108, 2013.

[35] F. Liu, S. Lo, C. Chuang et al., "Hot carriers in epitaxial graphene sheets with and without hydrogen intercalation: role of substrate coupling," Nanoscale, vol. 6, no. 18, pp. 10562-10568, 2014.

[36] F. Schedin, A. K. Geim, S. V. Morozov et al., "Detection of individual gas molecules adsorbed on graphene," Nature Materials, vol. 6, pp. 652-655, 2007.

[37] C. Chuang, Y. Yang, S. Pookpanratana, C. A. Hacker, C.-T. Liang, and R. E. Elmquist, "Chemical-doping-driven crossover from graphene to "ordinary metal" in epitaxial graphene grown on SiC", Nanoscale, vol. 9, no. 32, pp. 11537-11544, 2017.

[38] S. S. Datta, D. R. Strachan, E. J. Mele, and A. T. C. Johnson, "Surface Potentials and layer charge distributions in few-layer graphene films," Nano Letters, vol. 9, no. 1, pp. 7-11, 2009.

[39] C. Chuang, T.-P. Woo, F.-H. Liu et al., "High Current-Induced Electron Redistribution in a CVD-Grown Graphene Wide Constriction," Journal of Nanomaterials, vol. 2016, 7 pages, 2016.

[40] D. K. Ferry, Semiconductor Transport, Taylor \& Francis, London, UK, 2000.

[41] S. Vaziri, G. Lupina, C. Henkel et al., "A graphene-based hot electron transistor," Nano Letters, vol. 13, no. 4, pp. 1435-1439, 2013.

[42] D. R. Hang, C.-T. Liang, J.-R. Juang et al., "Electrically detected and microwave-modulated Shubnikov-de Haas oscillations in an $\mathrm{Al}_{0.4} \mathrm{Ga}_{0.6} \mathrm{~N} / \mathrm{GaN}$ heterostructure," Journal of Applied Physics, vol. 93, no. 4, pp. 2055-2058, 2003.

[43] J. R. Juang, T.-Y. Huang, T.-M. Chen et al., "Transport in a gated $\mathrm{Al}_{0.18} \mathrm{Ga}_{0.82} \mathrm{~N} / \mathrm{GaN}$ electron system," Journal of Applied Physics, vol. 94, no. 5, pp. 3181-3184, 2003. 


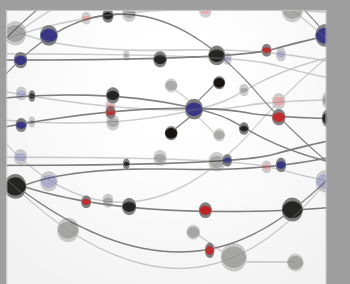

The Scientific World Journal
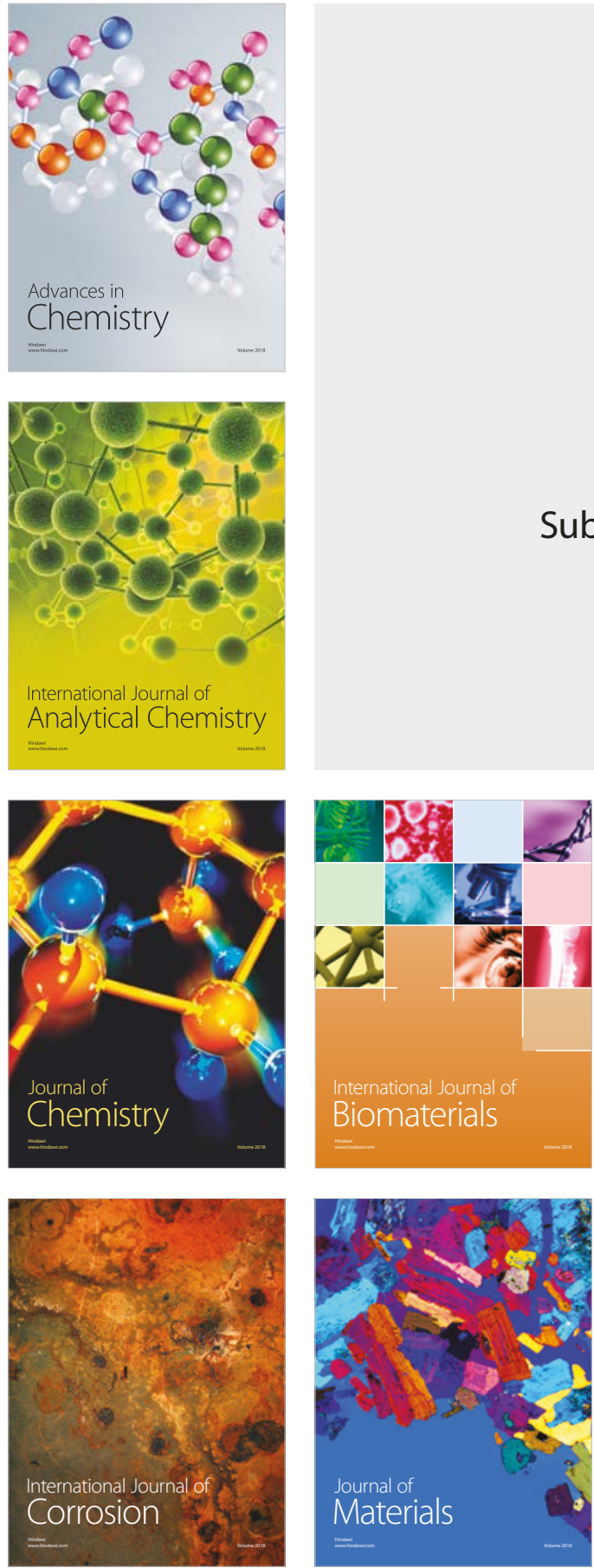

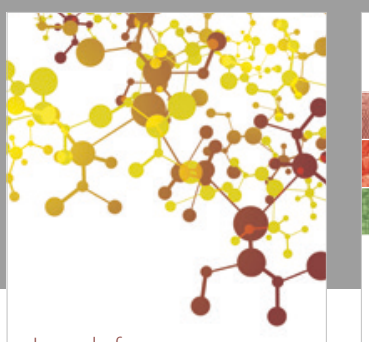

Journal of

Applied Chemistry
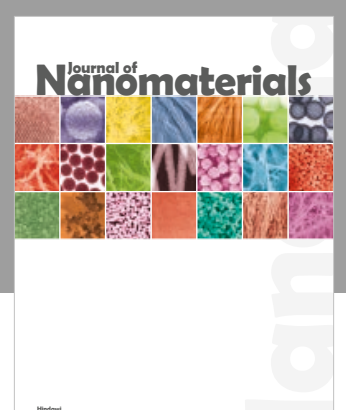

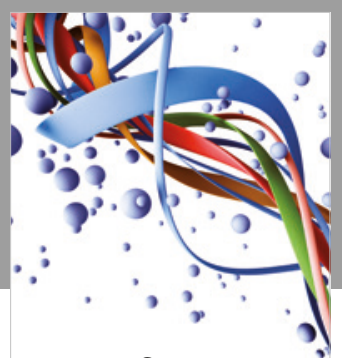

Scientifica

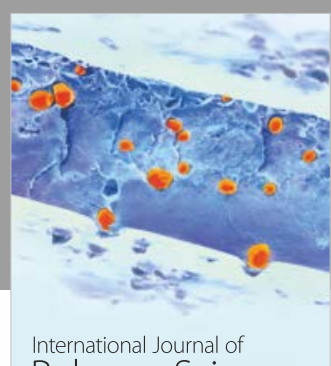

Polymer Science

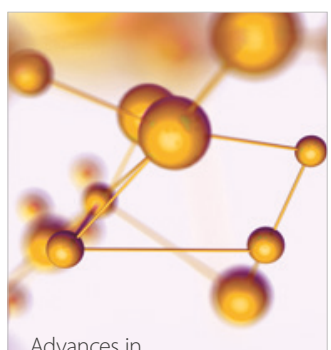

Physical Chemistry
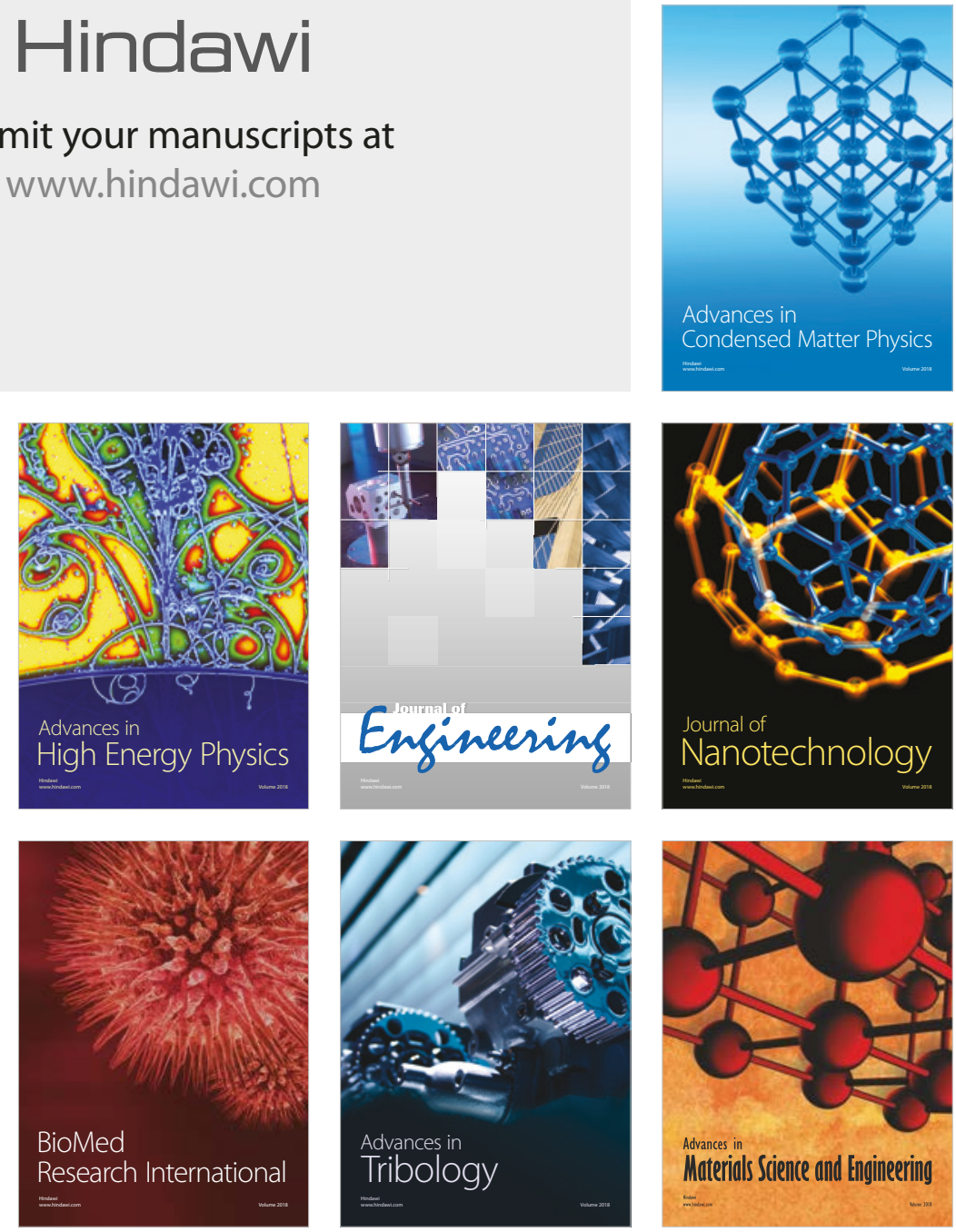